\title{
Hemodynamic changes in athletes' brains: is there any adaptation?
}

\author{
Egemen Manci ${ }^{1,3}$, Ozan C. Deniz ${ }^{2}$, Cagdas Guducu ${ }^{2}$, Erkan Gunay ${ }^{3}$ and Cem S. Bediz ${ }^{1,2,4}$ \\ ${ }^{1}$ Department of Physiology, Faculty of Medicine, Dokuz Eylül University, Izmir, Turkey \\ ${ }^{2}$ Department of Biophysics, Faculty of Medicine, Dokuz Eylül University, Izmir, Turkey \\ ${ }^{3}$ Faculty of Sport Science, Dokuz Eylül University, Izmir, Turkey \\ ${ }^{4}$ Faculty of Medicine, University of Kyrenia, Kyrenia, Cyprus
}

\begin{abstract}
This study compared the hemodynamic changes in the prefrontal cortex during sprint interval training (SIT) and recovery periods in sedentary and athletes. SIT was performed on a cycling ergometer on 12 male athletes and 9 sedentary participants. A functional near-infrared spectroscopy (fNIRS) device was used to record the hemodynamic changes of the prefrontal cortex throughout the protocol. The oxyhemoglobin $(\mathrm{Oxy}-\mathrm{Hb})$ levels in the prefrontal cortex were increased significantly, and the power outputs were decreased in repetitive Wingate anaerobic tests (WAnTs) in Sedentary and Athletes group $(p<0.001)$. In addition, the Sedentary group had higher Oxy-Hb values $(p<$ $0.001)$. However, the recovery times decreased significantly after all WAnTs $(p<0.05)$. Despite the increased fatigue, athletes performed better with less $\mathrm{Oxy}-\mathrm{Hb}$ than the sedentary participants. Also, the recovery of the Oxy-Hb values in the prefrontal region was faster in athletes. These results may highlight a possible brain adaptation in athletes.
\end{abstract}

Key words: Athlete's brain - Functional near-infrared spectroscopy - Brain hemodynamics Fatigue and recovery - Sprint interval training

Abbreviations: fNIRS, functional near-infrared spectroscopy; MP, mean power; Oxy-Hb, oxyhemoglobin; PFC, prefrontal cortex; PP, peak power; SIT, sprint interval training; WAnT, Wingate anaerobic test.

\section{Introduction}

"Mens sana in corpore sano" has been one of the most popular medical mottos since Juvenal's poem. As the Latin-speaking poet explained, without observing the exact mechanisms behind it, exercise affects the muscular and skeletal system and brain hemodynamics. As expected, the greatest effects of exercise can be seen among athletes, who are the participants of this research. An athlete can develop various chronic adaptations, such as improved maximum oxygen use capacity, decreased resting heart rate, hypertrophy, mitochondrial adaptation, increased antioxidant levels, and increased capillarization with a regular training program (Škof and Strojnik

Correspondence to: Egemen Manci, Department of Physiology, Dokuz Eylül University, Faculty of Medicine, 35320, Izmir, Turkey E-mail: egemenmanci@gmail.com
2006; Degens et al. 2019; Valenzuela et al. 2019). The physiologic adaptation of athletes is well known, but how brain adaptation develops and what features indicate the hemodynamics changes are less known. Hence, in recent studies, the question of "How does the brain structure and functioning of athletes change?" was discussed extensively (Belviranli et al. 2016; Herold et al. 2020b; Paruk et al. 2020). Considering the current knowledge, differences in various brain structures (amygdala, cerebellum, motor areas, gray matter), which can highlight brain plasticity, have been shown, especially in studies with well-trained athletes (Guo et al. 2017; Duru and Balcioglu 2018; Paruk et al. 2020). In addition to these studies, the effects of different exercise methods on some brain parameters (brain-derived neurotrophic factor, neuronal activity, tolerance to mental fatigue, cognitive performance) of athletes have been examined (Guo et al. 2017; Duru and Balcioglu 2018; Jaydari Fard et al. 2019; Seidel and Ragert

(c) The Authors 2021. This is an open access article under the terms of the Creative Commons Attribution-NonCommercial 4.0 International License (https://creativecommons.org/licenses/by-nc/4.0/), which permits non-commercial use, distribution, and reproduction in any medium, provided the original work is properly cited. 
2020). Furthermore, neural efficiency hypotheses were suggested by Haier et al. (1992). This hypothesis assumes that cognitive capacity drives the energy consumption of the brain. This consumption is also related to the activations of frontal areas (Rypma et al. 2002; Duns et al. 2014; Basten et al. 2015). Athletes also need to use many functions of the brain simultaneously during high-intensity workloads to perform successfully. Based on this ability, neuronal and hemodynamic efficiency plays a critical role. Therefore, the link between exercise type or intensity and cortical activations has to be established. The aforementioned studies suggest that exercise intensity can modulate cortical activations and athletes' brains work with lower neuronal loads at high exercise intensities, which can be assumed to be an indicator of neuronal efficiency. These results may highlight a possible brain adaptation in athletes. Hence, there has been growing interest in examining the oxygenation change in the prefrontal cortex (PFC) during exercise and clarifying the relationship between brain hemodynamics and exercise in the last decade (Monroe et al. 2016; Herold et al. 2019, 2020a). For this purpose, we also employed sprint interval training (SIT) as a fatiguing exercise protocol to show how the volunteers handled demanding tasks and how the hemodynamic parameters changed in this process.

In this context, repeated sprints as an SIT method, which is a popular exercise program, is frequently used in the development of athletes. Especially in team sports, reaching and maintaining high speeds (endurance in speed) plays a critical role during games. High physiologic stress occurs and it is essential to improve the anaerobic energy production capability of the muscle and neuromuscular efficiency to meet these demands. High-intensity interval training (HIIT) SIT is widely used in the development of this key factor. In the SIT model, active rest or passive rest periods can be used in the range of 1-4 min at low loads between 'all-out' loads performed between 20-45 s (Buchheit and Laursen 2013a, 2013b) Practices with active rest periods are preferred in SIT programs performed with a bicycle ergometer (Milanovic et al. 2015; Vollaard and Metcalfe 2017; Kujach et al. 2018).

Fatigue and recovery are complex mechanisms starting from the central nervous system, including the muscle cells themselves. In this context, the most important marker in determining athletes' performances and identifying elite athletes are the conditions of fatigue and recovery. As a result, monitoring the fatigue and recovery process with various markers (subjective, heart rate, biochemical, neuromuscular, and performance markers) or using many tools (Delayedonset muscle soreness (DOMS) scale, jump test, blood lactate levels) are frequently used in this area and a practical method is sought as standard (Kent-Braun 1999; Škof and Strojnik 2006; Wiewelhove et al. 2015). Alternatively, the reason for the failure of the neurovascular system during fatigue is tried to be explained by central and peripheral factors (Kent-Braun
1999; Monroe et al. 2016). Although there are many fatigue and recovery markers (peripheral) in the field of sports sciences and still being researched, hitherto there are no such exercise and fatigue markers concerning brain hemodynamics. In one of the important approaches to fatigue, the brain is responsible for controlling, regulating, and finishing the processes, if needed. This hypothesis is called the "central governor" hypothesis, in which fatigue is entirely dependent on the central system of the body. Therefore, observing the central changes during fatigue is suggested (Weir et al. 2006). One of the most promising tools for observing these central changes is functional near-infrared spectroscopy (fNIRS). This is an easy-to-use and noninvasive optical neuroimaging technique based on the theory of neurovascular coupling and optical spectroscopy. It is frequently used for monitoring brain hemodynamics changes that occur during exercise or simpler motor tasks (Herold et al. 2018; Güdücü and Bediz 2019; Günay et al. 2019). In studies, the PFC has been monitored due to its importance in the processes of sustainability exercise. Also, the regulation of oxygenation may change due to the brain adaptation or plasticity of athletes.

To our knowledge, the relationship between fatigue and recovery concerning the PFC hemodynamic changes of athletes and sedentary individuals during exercise are not fully understood. Also, the adaptation of athletes' brains and possible mechanisms have not been enlightened in previous studies. Therefore, the present study aimed to compare the hemodynamic changes and responses of the PFC in sedentary individuals and athletes during SIT and SIT recovery periods to reveal possible brain adaptations.

\section{Materials and Methods}

\section{Participants and experimental design}

Twelve male athletes (basketball players, age: $21.30 \pm 1.49$ year) who regularly trained 5 days per week for at least 10 years (Athletes group) and 9 sedentary males (age: 23.00 \pm 1.41 years) who did not exercise regularly and had a daily physical activity level of 1.5-3 metabolic equivalent tasks (METs) according to the International Physical Activity Questionnaire (Sedentary group) were included in the study. The exclusion criteria were neurologic, medical or cardiovascular diseases and use of medication. Additionally, we excluded players who experienced an injury and did not train in the last season. The sample size was calculated with the GPower 3.1.9.4 programme. Accordingly, the sample size was described as 12 participants with an alpha (mistake) rate of $5 \%$ and $70 \%$ power with the medium effect size for two groups of repeated measures. All measurements were held between 10:30-12:00 a.m. and participants were asked not to consume any food and drink (e.g, caffeine, alcohol, vitamin 


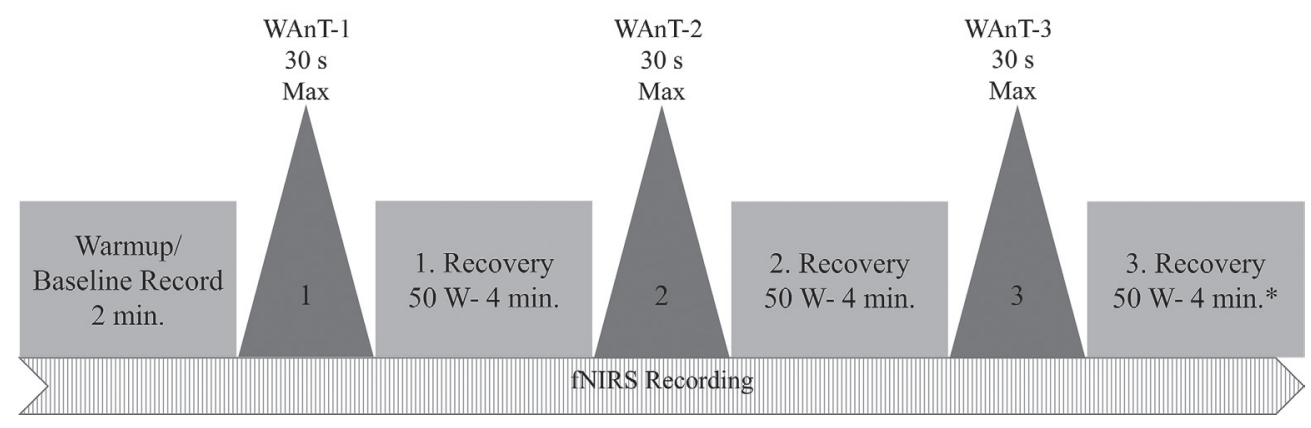

Figure 1. Experimental procedure of the functional near-infrared spectroscopy (fNIRS) recording. Wingate anaerobic test (WAnT), replicated for three times (WAnT1, WAnT-2, WAnT-3). * In the last repetition, 2 min were recorded instead of $4 \mathrm{~min}$ due to the excessive fatigue of the sedentary participants.

complexes) that could affect their performance, $2-3 \mathrm{~h}$ before the exercise. All participants were informed about the procedures (Fig. 1) and each gave written consent. Measurements were taken in Dokuz Eylül University Faculty of Medicine, Exercise Physiology Laboratory and Human Factor Laboratories of Biophysics Department. The participants' body compositions were analyzed using a laboratory-type InBody 720 (Body Composition Analyzer, South Korea) bioelectric impedance device. The participants' heights were measured using a stadiometer while they were barefoot (G-Tech International, Korea). The study was approved by the Local Ethics Committee of Dokuz Eylül University (2016/18-24). The study also conformed to the standards set out in the Declaration of Helsinki.

\section{Exercise protocol}

First, the exercise protocol was introduced to the participants, then they were seated on the cycle-ergometer and started a 4-min warm-up at a power of 50 Watts (W) with a pedaling speed of $60-70 \mathrm{rpm}$. In the SIT protocol, the Wingate anaerobic test (WAnT) has been employed for sprint training. During the warm-up, the participants were requested to sprint twice to determine their maximum pedaling rate for the WAnT and these rates were recorded for each participant. WAnT, which is an exhaustive (all-out) test for determining anaerobic performance, was applied to the participants three times (WAnT-1, WAnT-2, WAnT-3) with $4 \mathrm{~min}$ of active recovery between the repetitions using a cycling ergometer (Monark 839E, Sweden) (Dotan and Bar-Or 1983; Naves et al. 2018). During the WAnT, the participants were asked to use their maximum effort concerning pedaling at a maximum speed for $30 \mathrm{~s}$, against a load ( $80 \mathrm{~g}$ b.w. and $60 \mathrm{~g}$ b.w. for the athletes and sedentary individuals, respectively) (Weinstein et al. 1998). Then, the participants pedaled for $4 \mathrm{~min}$ at a speed of $60-70 \mathrm{rpm}$ at $50 \mathrm{~W}$ during the recovery periods, in which no exhaustive exercise was applied. The total time for the whole exercise protocol was $15 \mathrm{~min}$ and $30 \mathrm{~s}$, excluding the warm-up period (Fig. 1). The mean power (MP) and peak power (PP) outputs of the participants were recorded during the WAnT. fNIRS was used to record the brain hemodynamics in the whole experimental protocol (as shown in Fig. 1).

\section{fNIRS recordings}

In this study, an fNIRS device, which is non-invasive and easy to apply (fNIR Devices 1100 LLC, MD, USA), was used to record hemodynamic changes in the PFC. The sensor pad of the device was placed on the participants' forehead as previously described (Bediz et al. 2016; Ayaz et al. 2019), in which the position of the sensor pad corresponds to the PFC according to the user manual of the device. Also, the pad was covered with a black band to reduce ambient light. Sixteen optodes recorded the hemodynamics at a frequency of $2 \mathrm{~Hz}$ with a $2.5-\mathrm{cm}$ source-detector separation. The amount of light sent from a light source and perceived by sensors is examined, and the oxy-hemoglobin (Oxy-Hb) concentration changes in that region are calculated over the amount of light absorption in the tissues. The penetration depth of the infrared light sent by the fNIRS device is around $3 \mathrm{~cm}$ and it can reach the cortical regions. The differential pathlength factor (DPF) we are using is about 6 (specifically $1000 \times 0.015 / 2.5)$. The calculations and analysis were executed using the Cognitive Optical Brain Imager (COBI) software. During the off-line analysis, the raw fNIRS data were filtered using a band-pass finite-impulse response (FIR) filter with a range of $0.01-0.50 \mathrm{~Hz}$ to eliminate possible respiratory and heart rate-related signals and unrelated high-frequency noise using fNIRSoft (Huppert et al. 2009; Ayaz et al. 2012; Pinti et al. 2018). Also, to overcome the physiological noise effects, we employed a common average reference (CAR) method which is described by Pfurtscheller et al. (2010) and von Lühmann et al. (2020) and re-calculated the whole data accordingly. Basically, we calculated the mean of all channels in every single time point and subtracted it from every single channel. The fNIRS recordings were baselined according to the pre-WAnT period, in which the participants were pedalling without any load for $2 \mathrm{~min}$. Then, the Oxy-Hb average of both groups during active rest and WAnTs were calculated. Additionally, the time taken for the participants to reach the maximum $\mathrm{Oxy}-\mathrm{Hb}$ values was calculated using frames. 


\section{Statistical analysis}

The SPSS 22 program was used for statistical evaluations. The statistical distribution type of the data was evaluated using normality tests (Kolmogorov-Smirnov). According to these test results, it was observed that the data group did not have a normal distribution. Therefore, the Friedman test was used for multiple comparisons. The MannWhitney $\mathrm{U}$ and Wilcoxon test with Bonferroni correction was used for pairwise comparisons. The significance level was set at $p<0.05$ and the effect sizes were reported as partial $\eta^{2}$.

\section{Results}

\section{Demographic and physiologic parameters}

Twelve male basketball players (mean age: $21.30 \pm 1.49$ years; mean height: $184.6 \pm 8.34 \mathrm{~cm}$; mean weight: $83.5 \pm 6.27 \mathrm{~kg}$ ), who trained 5 days per week for at least 10 years, and nine sedentary male participants (mean age: $23.00 \pm 1.41$ years; mean height: $182.0 \pm 7.12 \mathrm{~cm}$; mean weight: $81.6 \pm 9.24 \mathrm{~kg}$ ), without an exercise regime, participated in the study.

\section{Power outputs}

A significant difference was revealed when the PP values of the athletes during repeated WAnT's were compared using the Friedman test $(p<0.001)$. Comparisons of WAnT's with each other by employing the Wilcoxon test, there was a significant decrease between WAnT-1 (860.57 W) and WAnT-3 (725.50 W), and between WAnT-2 (828.99 W) and WAnT-3 (Fig. 2A; $p=0.004, \eta^{2}=0.764$ and $p=0.002$, $\eta^{2}=0.879$, respectively). There was no significant difference between WAnT-1 and WAnT-2. Based on these data, it can be said that the PP values of athletes gradually decreased after each WAnT.

A statistically significant difference was found between the athletes' MP values of repeated WAnT using the Friedman test $(p<0.001)$. According to the pairwise comparisons, which were performed using the Wilcoxon test, there were significant differences between WAnT-1 (625.47 W) and WAnT-3 (516.92 W), and between WAnT-2 (560.03 W) and WAnT-3 (Fig. 2B; $p=0.002, \eta^{2}=0.229$ and $p=0.005, \eta^{2}=$ 0.728 , respectively). These analyses revealed a significant decrease in the MP values as the repetition occurred. However, there was no significant difference between WAnT-1 and WAnT-2 $(p=0.023)$.

When the PP and MP values of the sedentary individuals during repeated WAnT's were compared using the Friedman test, a significant difference was observed $(p<0.05)$. However, this significant difference disappeared in the pairwise comparisons using the Wilcoxon test (with Bonferroni correction). These results show that although the sedentary participants exerted a certain level of power during the WAnT's, there was no difference between repetitions (Fig. 2).

PP and MP values were compared using a betweengroup design. According to these comparisons, the athletes achieved significantly higher power outputs in all repetitions
A

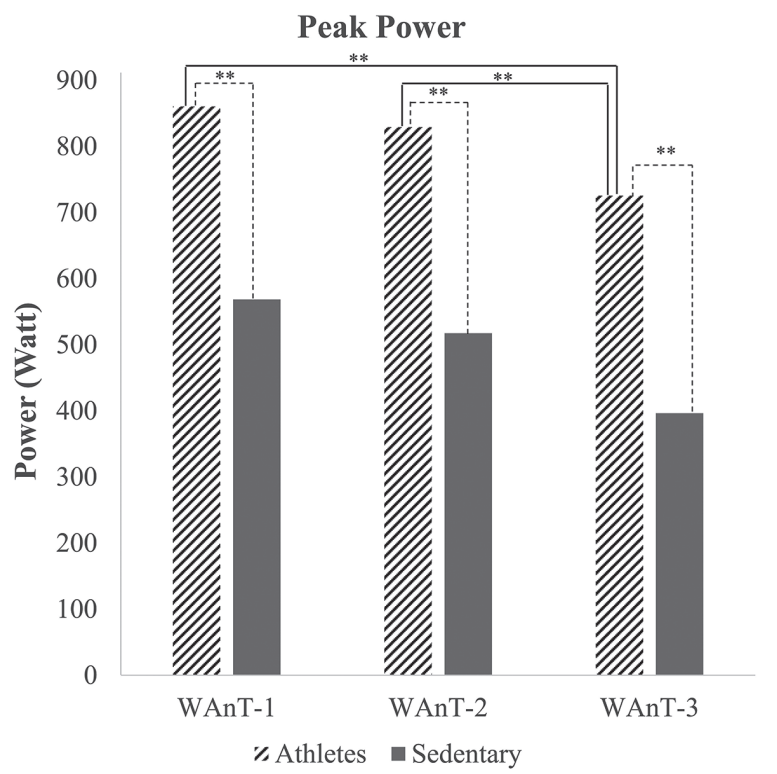

B

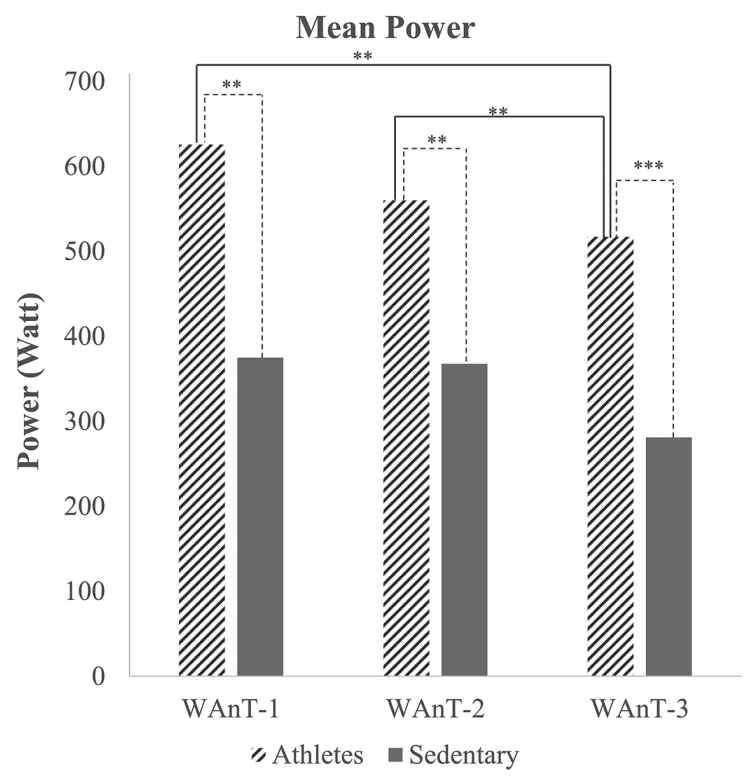

Figure 2. Peak Power (A) and Mean Power (B) values of Athletes and Sedentary groups during the WAnT. Dashed lines show comparisons between groups, straight lines represent within-group comparisons. ${ }^{* *} p<0.01,{ }^{* * *} p<0.001$. 


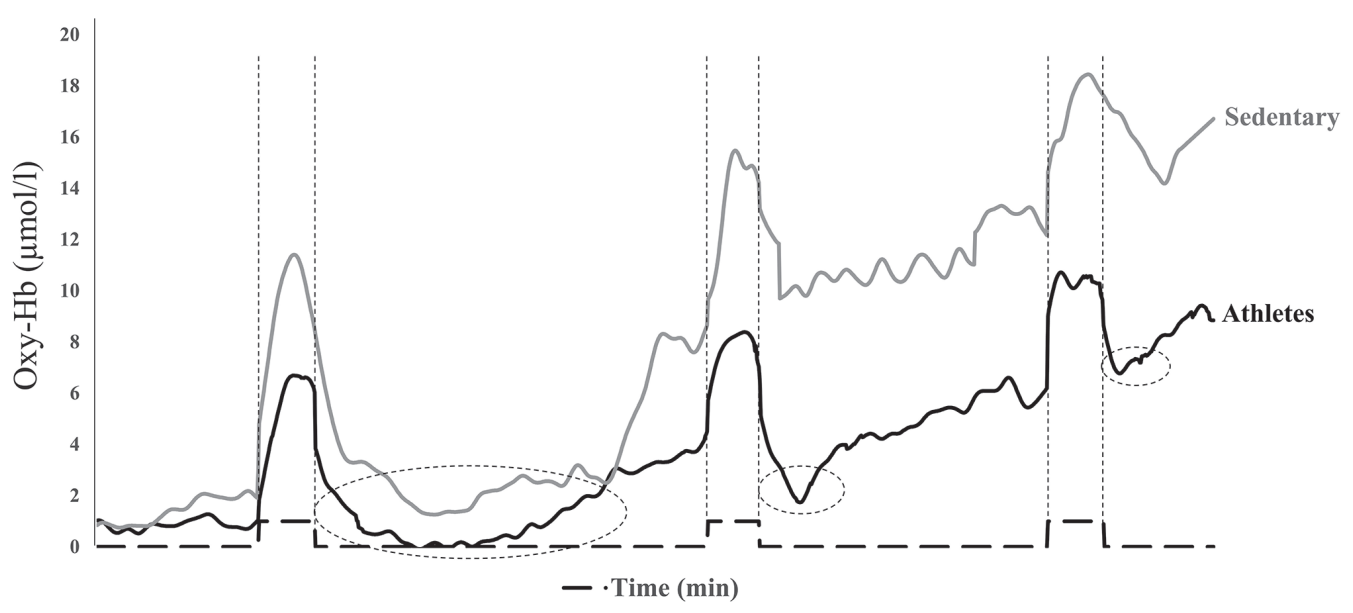

Figure 3. Comparison of Oxy-Hb data of the Athletes and Sedentary groups. Dashed lines show the WAnT periods. Circular areas are describing recovery $\mathrm{Oxy}-\mathrm{Hb}$ values, in which the $\mathrm{Oxy}-\mathrm{Hb}$ values are returning to the pre-WAnT values, of the participants after each WAnT.

(PP values (Fig. 2A, dashed lines): WAnT-1: $p=0.002, \eta^{2}=$ 0.228 ; WAnT-2: $p=0.002, \eta^{2}=0.288$; WAnT-3: $p=0.001$, $\eta^{2}=0.324$. MP values (Fig. $2 \mathrm{~B}$, dashed lines): WAnT- $1: p=$ $0.002, \eta^{2}=0.288$; WAnT-2: $p=0.002, \eta^{2}=0.288$; WAnT-3: $\left.p<0.001, \eta^{2}=0.324\right)$.

\section{Hemodynamic parameters}

Oxy-Hb values were significantly higher during each WAnT compared with the previous WAnT $(p<0.001)$. In addition, it was observed that the $\mathrm{Oxy}-\mathrm{Hb}$ values of the sedentary group were significantly higher than in the athletes (Fig. 3, $p<0.001$, for all). When the recovery data of athletes and sedentary individuals were compared, the Oxy-Hb values during the recovery periods of the sedentary group were also significantly higher (Fig. 3, $p<0.001$, for all).

Another time-related analysis was performed concerning the time when the two groups reached their maximum Oxy-Hb level, which is described as $\operatorname{Tmax}_{\mathrm{OxyHb}}$, during the WAnTs (Fig. 4). A significant shortening was revealed in the times according to the within-group triple comparisons performed using the Friedman test $(p<0.05)$.

In the pairwise comparisons for the athlete group, there was a significant difference between WAnT-1-Tmax $\mathrm{x}_{\mathrm{OxyHb}}$

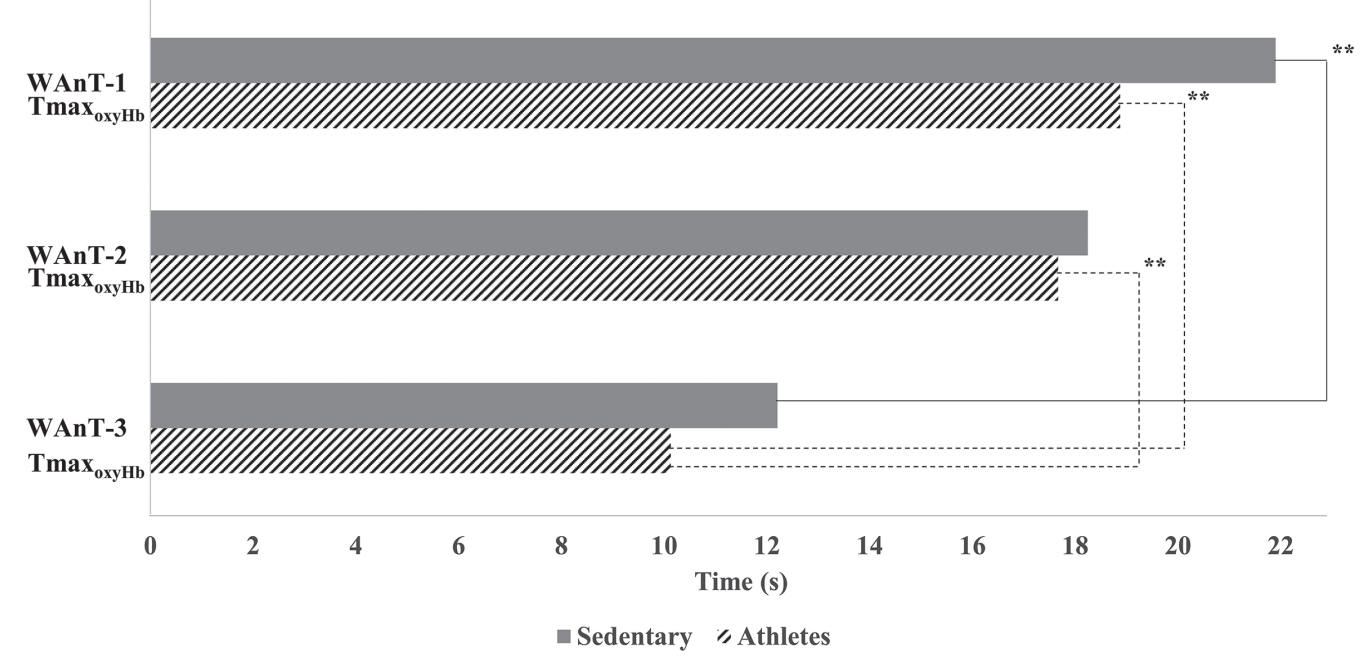

Figure 4. Maximal Oxy-Hb reaching times (WAnT-Tmax ${ }_{\mathrm{OxyHb}}$ ) in Athletes and Sedentary group. WAnT-Tmax oxyHb $_{\text {is }}$ describing the time to reach the participants' maximum Oxy-Hb levels. These durations for each recovery has been demonstrated. Dashed lines point out the significant differences between the WAnT sessions for the Athletes group while the continuous lines point out the significant differences between the WAnT sessions for the Sedentary group. ${ }^{* *} p<0.01$. 
(18.87 s) and WAnT-3-Tmax ${ }_{\mathrm{OxyHb}}(10.12 \mathrm{~s})$, and between WAnT-2-Tmax ${ }_{\text {oxyHb }}\left(17.66 \mathrm{~s}\right.$ ) and WAnT-3 Tmax OxyHb $_{\text {(both }}$ $\left.p=0.003, \eta^{2}=0.505\right)$, but there was no significant difference between WAnT-1-Tmax $x_{\mathrm{oxyHb}}$ and WAnT-2-Tmax ${ }_{\mathrm{oxy} H \mathrm{H}}$.

In the sedentary group, a significant shortening was observed only between WAnT-1 (21.90 s) and WAnT-3 (12.20 s; $\left.p=0.004, \eta^{2}=0.496\right)$. In the between-group analysis, no significant difference was revealed.

Despite the similar Oxy-Hb values of both groups before the exercise, this pattern was differentiated during the WAnTs. In particular, Oxy- $\mathrm{Hb}$ values had a rapid increase and decrease in athletes. This decrement led to lower $\mathrm{Oxy}-\mathrm{Hb}$ values than its pre-exercise values. The duration between the Oxy-Hb values of the participants after WAnT recovering to pre-WAnT values (WAnT Trec ${ }_{\text {oxyHb }}$ ) was calculated (Fig. 3, the circled area).

However, in the sedentary group, the Oxy-Hb values did not decrease after the WAnT and stayed higher than the pre-exercise values. Therefore, WAnT Trec ${ }_{0 x y H b}$ values could not be calculated for the sedentary group. According to our results, the athletes' recovery times after all WAnTs (WAnT-1: $148 \mathrm{~s}$; WAnT-2: $72 \mathrm{~s}$; WAnT-3: $23 \mathrm{~s}$ ) decreased significantly ( $p<0.001$; Fig. 5).

\section{Discussion}

In this study, power outputs and hemodynamic changes in the PFC during repetitive maximal sprint exercise were evaluated in athletes and sedentary participants using fNIRS. In previous studies, knowledge about the possible effects of different types of exercise on brain hemodynamics was used to determine an optimal exercise for brain health, and prescribing exercise as an important future topic (Herold et al. 2019, 2020b). However, concerning the oxygenation of the PFC, the difference or relationship between the PFC hemodynamics of sedentary individuals and athlete's has been investigated in an insignificant number of studies. Therefore, in this study, hemodynamic changes in the PFC of athletes and sedentary groups were evaluated during a high-intensity exercise protocol using fNIRS.

The power output of the athletes was much higher than among the sedentary participants. However, the power output similarly changed with the repetitions in both groups (Fig. 2). The fact that the power outputs with every WAnT repetition decreased in both groups may be considered as a maximum effort and possible fatigue indicator of the participants.

On the frontal hemodynamic aspect, Oxy-Hb values were significantly higher in both groups during each WAnT than in the previous WAnT (Fig. 3), which is consistent with a previous study by Monroe et al. (2016). Additionally, the sedentary group had higher $\mathrm{Oxy}-\mathrm{Hb}$ values than the athletes. This could support the idea that the physiologic adaptations of the athletes were not limited to the cardiovascular (lower heart rate and higher performance) system, they might also be valid for brain hemodynamics. Consequently, athletes may have better regulation of $\mathrm{Oxy}-\mathrm{Hb}$, which could be considered a sign of hemodynamic plasticity.

A similar approach was also proposed by Wu et al. (2017) in a study in which the authors stated that individuals showed more efforts to avoid the effects of fatigue. The PFC is especially responsible for this process. In the present study, it can be assumed that the athletes tried to be ready for the next sprint and therefore their prefrontal oxygenation increased after each sprint. The difference between the

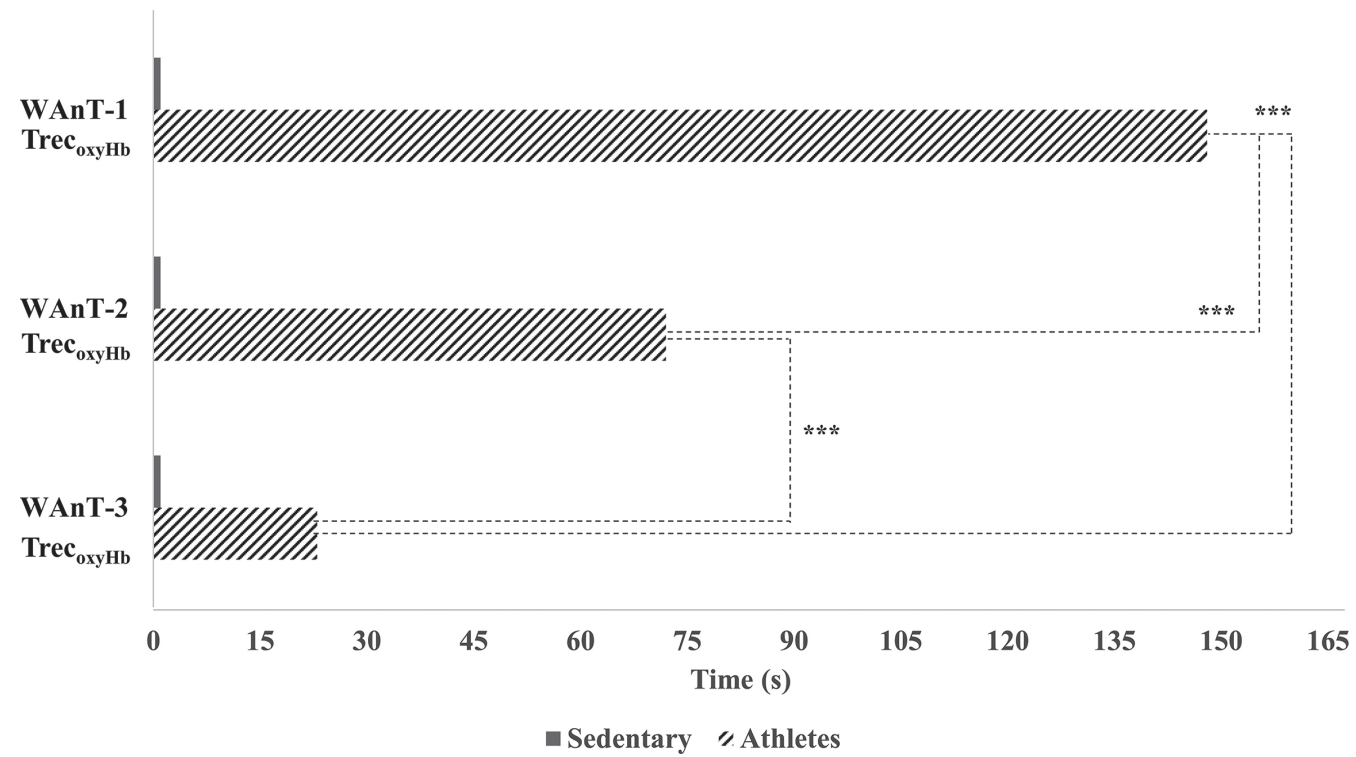

Figure 5. Oxy-Hb recovery times (WAnT$\mathrm{Trec}_{\text {oxyHb }}$ ) after WAnT in Athletes group. WAnT$\mathrm{Trec}_{\mathrm{oxyHb}}$ is describing the duration between the Oxy-Hb values of the participants after WAnT recovering to pre-WAnT values. Dashed lines point out the significant differences between the WAnT sessions for the Athletes group. ${ }^{* *} p<$ 0.001 . 
Oxy-Hb values of the athletes and sedentary participants may indicate possible neural efficiency. In this context, basketball can be assumed as a complex sports branch in which neuronal activations would be higher and, due to this higher activity, neural efficiency can be discriminated between the groups.

Hence, many studies are reporting structural and functional changes in elite athletes (Hänggi et al. 2010; Di et al. 2012; Duru et al. 2018). One of these studies indicated that elite Karate athletes had increased gray matter volumes in the temporal, occipital, and premotor areas of the brain. Additionally, greater white matter volumes in the hypothalamus and increased connectivity between the areas of visual perception and motor planning have been reported (Duru et al. 2018). They connect these changes to the many years of the specialized training sessions. Neuronal mechanisms were investigated using functional magnetic resonance imaging (fMRI) in elite, professional, and amateur athletes by Kim et al. (2014) who reported that elite athletes had higher cortical activation in the supplementary motor areas and cerebellum. In the present study, PFC oxygenation was investigated with another aspect. The findings related to the $\mathrm{Oxy}-\mathrm{Hb}$ had similarities to previous studies. We thought that the difference between the athletes and sedentary participants was mostly due to the many years of training. This experience may positively change the athletes' brains, which leads them to preserve energy if they can. In other words, athletes may have developed an energy consumption strategy in their PFC.

In another study, neurovascular connectivities were examined in endurance athletes and physically active adults regarding the primary motor cortex. Despite the insignificant differences between them as determined using neural efficiency, the authors also stated that athletes did the same work with reduced PFC activation compared with active adults (Seidel et al. 2019).

Another study indicated a decreased brain activity in table-tennis players under the task of visuo-spatial tasks related with sports and not related with sports. Meier et al. (2016) suggested the brain adaptation is specific to the sports and these adaptations observable only in specific areas related with the specific skills. They demonstrated that on handball players by employing the MRI. According to that study, the gray matter volumes are increased in hand related motor areas. Another evidence is spotted with dancers. Again, an increase in gray matter volumes of foot related motor areas had been spotted. These studies showed changes in brain structures which are occurred due to the chronic effects of specified training to be a professional athlete. In the present study we believe that the changes in PFC could indicate a functional adaptation as well as the structural adaptation. Further studies are needed to show both changes at the same time.
In another analysis, the time to reach the maximum Oxy-Hb level during repeated WAnTs was determined in the Athletes and Sedentary groups. Accordingly, only the athlete group's time was shortened as the number of repetitions increased. These results may also suggest an adaptation of athletes, who can improve their vasodilation mechanisms and prepare themselves for the next challenging exercise task by delivering $\mathrm{Oxy}-\mathrm{Hb}$ to the prefrontal region in a shorter time. Thus, the athletes perform better in this type of exercise.

According to the analysis of Oxy-Hb values in the recovery periods, the Oxy-Hb values of both groups were increased compared with the previous WAnTs. However, only the increase in the sedentary group was statistically significant. This might be the result of increasing fatigue with repeated supramaximal exercise and the changes in the hemodynamic response of recovery. These changes may suggest a compensation mechanism in brain responses related to fatigue. However, further studies are needed to clarify this hypothesis. Monroe et al. (2016) reported a large increase in Oxy-Hb in the dorsolateral PFC during a sprint and active recovery in the data they received from four channels. They also revealed that the $\mathrm{Oxy}-\mathrm{Hb}$ values were still increasing during the fourth repetition of the sprint despite the increased fatigue. This increase was also in line with our $\mathrm{Oxy}-\mathrm{Hb}$ findings. In addition, our analyses showed that the Oxy-Hb data at the end of the WAnTs had fallen below the initial levels of the exercise and even below the baseline values during the first WAnT. This trend is similar to other studies (Monroe et al. 2016; Günay et al. 2019). Rebounding to the starting levels and even exceeding them after reaching the nadir may denote a supercompensation mechanism, which creates an Oxy-Hb roller-coaster/fluctuation. Although fatigue and power values increase with each repetition, recovery in the prefrontal region becomes faster (Fig. 5). These faster processes might be accepted as an important indicator of the brain adaptations of athletes and it should be supported by further studies to become a parameter used in determining the levels of elite athletes.

The sedentary brain may develop a saving strategy by collecting higher levels of oxygen to sustain high-intensity exercise stress. As the brain specializes in exercise, it may need less Oxy-Hb use. In the rest periods between sprints, the same protection behavior continues in the sedentary brain, whereas the process of returning to normal values starts in athletes. Could a decrease in Oxy- $\mathrm{Hb}$ in the brain under physical stress be a marker for high performance? Exponential increases may represent a protective behavior and poor performance, and moderately controlled increases may represent a positive response (high-performance output) due to adaptation to stress. How these changes occur in the PFCs of athletes and sedentary brains under exercise and how this situation can be explained and used in personalized exercise 
prescriptions are issues that remain to be resolved (Herold et al. 2019, 2020b).

One of the most important limitations of this study was the inability to compare the same recovery parameters with sedentary groups. In further studies, it will be important to examine how the recovery periods change after WAnTs by applying the same exercise protocol to wider groups. Another important limitation is that heart rate, Deoxy- $\mathrm{Hb}$, and total hemoglobin data could not be evaluated during the study. Also, including whole-head fNIRS recordings would be an essential contribution to future studies. Furthermore, we could not compare the same participant size for the groups due to the Covid-19 pandemic regulations. In the future studies, this group size should be enlarged accordingly. Another limitation can be the data analysis of fNIRS. In the recent guidelines (Herold et al. 2017; Menant et al. 2020; Yücel et al. 2021), there are various methods suggested for the rejection of motion related artifacts. In this present study, we did not prefer to apply these methods, since we compared the repetitive situations of the WAnTs. In the future studies the efficiency of the proposed or suggested methods can be proved under the similar exercise protocols.

\section{Conclusion}

In this study, hemodynamic changes in the PFC during WAnT periods were observed in athletes and sedentary individuals. Consequently, the time to reach the maximum Oxy-Hb levels in the two groups, Oxy-Hb parameters during the whole exercise, and additionally the recovery periods of athletes were examined. According to these results, athletes' vasodilation mechanisms in the PFC work faster than in sedentary individuals to meet oxygen needs, and they also perform better with less Oxy-Hb than sedentary individuals. This shows that there is a physical difference between athletes and sedentary people and differences in terms of brain adaptation.

Although repeated high-intensity exercise decreases strength due to muscular and central fatigue, the hemodynamic increase seen in $\mathrm{Oxy}-\mathrm{Hb}$ in the brain anterior region increases the activities of the executive centers in the PFC to compensate for the decreased exercise capacity. In addition, these results may be evidence that the mechanism works better in athletes. In further studies, it is planned to perform physiologic measurements similarly with special experimental setups to illuminate the structures that cause different hemodynamics, and the parameters and markers that make athletes' brains special.

Conflict of interest. The authors have no conflicts of interests or personal relationships that are directly relevant to the content of this article.
Acknowledgements. We would like to thank all the participants in the study. We would also like to thank Emre Eskicioglu for his contribution to the previous version of the manuscript.

Funding. This research did not receive any specific grant from funding agencies in the public, commercial, or not-for-profit sectors.

\section{References}

Ayaz H, Izzetoglu M, Izzetoglu K, Onaral B (2019): The use of functional near-infrared spectroscopy in neuroergonomics. In: Neuroergonomics. pp. 17-25, Elsevier https://doi.org/10.1016/B978-0-12-811926-6.00003-8

Ayaz H, Shewokis PA, Bunce S, Izzetoglu K, Willems B, Onaral B (2012): Optical brain monitoring for operator training and mental workload assessment. Neuroimage 59, 36-47 https://doi.org/10.1016/j.neuroimage.2011.06.023

Basten U, Hilger K, Fiebach CJ (2015): Where smart brains are different: A quantitative meta-analysis of functional and structural brain imaging studies on intelligence. Intelligence 51, 10-27 https://doi.org/10.1016/j.intell.2015.04.009

Bediz CS, Oniz A, Guducu C, Ural Demirci E, Ogut H, Gunay E, Cetinkaya C, Ozgoren M (2016): Acute supramaximal exercise increases the brain oxygenation in relation to cognitive workload. Front. Hum. Neurosci. 10, 174 https://doi.org/10.3389/fnhum.2016.00174

Belviranli M, Okudan N, Kabak B, Erdoğan M, Karanfilci M (2016): The relationship between brain-derived neurotrophic factor, irisin and cognitive skills of endurance athletes. Phys. Sportsmed. 44, 290-296 https://doi.org/10.1080/00913847.2016.1196125

Buchheit M, Laursen PB (2013a): High-intensity interval training, solutions to the programming puzzle. Sports Med. 43, 313-338 https://doi.org/10.1007/s40279-013-0029-x

Buchheit M, Plews DJ, Laursen PB, Stanley J, Kilding AE (2013b): Training adaptation and heart rate variability in elite endurance athletes: opening the door to effective monitoring. Sports Med. 43, 773-781 https://doi.org/10.1007/s40279-013-0071-8

Degens H, Stasiulis A, Skurvydas A, Statkeviciene B, Venckunas T (2019): Physiological comparison between non-athletes, endurance, power and team athletes. Eur. J. Appl. Physiol. 119, 1377-1386 https://doi.org/10.1007/s00421-019-04128-3

Di X, Zhu S, Jin H, Wang P, Ye Z, Zhou K, Rao H (2012): Altered resting brain function and structure in professional badminton players. Brain Connect. 2, 225-233 https://doi.org/10.1089/brain.2011.0050

Dotan R, Bar-Or O (1983): Load optimization for the Wingate Anaerobic Test. Eur. J. Appl. Physiol. Occup. Physiol. 51, 409-417 https://doi.org/10.1007/BF00429077

Dunst B, Benedek M, Jauk E, Bergner S, Koschutnig K, Sommer M, Neubauer AC (2014): Neural efficiency as a function of task demands. Intelligence 42, 22-30 https://doi.org/10.1016/j.intell.2013.09.005

Duru AD, Balcioglu TH (2018): Functional and structural plasticity of brain in elite karate athletes. J. Healthc. Eng. 2018, 8310975 
https://doi.org/10.1155/2018/8310975

Guo Z, Li A, Yu L (2017): „Neural efficiency“ of athletes“ brain during visuo-spatial task: an fMRI study on table tennis players. Front. Behav. Neurosci. 11, 72 https://doi.org/10.3389/fnbeh.2017.00072

Güdücü C, Bediz CS (2019): The relationship between the performance and brain oxygenation during acute supramaximal exercise. Turk. J. Sports Med. 54, 242-249 https://doi.org/10.5152/tjsm.2019.138

Gunay E, Guducu C, Bediz CS (2019): How does isometric exercise affect the haemodynamics of the brain? Neurol. Sci. Neurophysiol. 36, 33-37 https://doi.org/10.5152/NSN.2019.10844

Haier RJ, Siegel B, Tang C, Abel L, Buchsbaum MS (1992): Intelligence and changes in regional cerebral glucose metabolic rate following learning. Intelligence 16, 415-426 https://doi.org/10.1016/0160-2896(92)90018-M

Hänggi J, Koeneke S, Bezzola L, Jäncke L (2010): Structural neuroplasticity in the sensorimotor network of professional female ballet dancers. Hum. Brain Mapp. 31, 1196-1206 https://doi.org/10.1002/hbm.20928

Herold F, Wiegel P, Scholkmann F, Thiers A, Hamacher D, Schega L (2017): Functional near-infrared spectroscopy in movement science: a systematic review on cortical activity in postural and walking tasks. Neurophotonics 4, 041403 https://doi.org/10.1117/1.NPh.4.4.041403

Herold F, Wiegel P, Scholkmann F, Muller NG (2018): Applications of functional near-infrared spectroscopy (fNIRS) neuroimaging in exercise-cognition science: A systematic, methodologyfocused review. Clin. Med. 7, 466 https://doi.org/10.3390/jcm7120466

Herold F, Muller P, Gronwald T, Mueller NG (2019): Dose-response matters! A perspective on the exercise prescription in exercisecognition research. Front. Psychol. 10, 2338

https://doi.org/10.3389/fpsyg.2019.02338

Herold F, Aye N, Lehmann N, Taubert M, Muller NG (2020a): The contribution of functional magnetic resonance imaging to the understanding of the effects of acute physical exercise on cognition. Brain Sci. 10, 175 https://doi.org/10.3390/brainsci10030175

Herold F, Gronwald T, Scholkmann F, Zohdi H, Wyser D, Muller NG, Hamacher D (2020b): New directions in exercise prescription: is there a role for brain-derived parameters obtained by functional near-infrared spectroscopy? Brain Sci. 10, 342 https://doi.org/10.3390/brainsci10060342

Huppert TJ, Diamond SG, Franceschini MA, Boas DA (2009): HomER: a review of time-series analysis methods for nearinfrared spectroscopy of the brain. Appl. Opt. 48, 280-298 https://doi.org/10.1364/AO.48.00D280

Jaydari Fard S, Tahmasebi Boroujeni S, Lavender AP (2019): Mental fatigue impairs simple reaction time in non-athletes more than athletes. Fatigue 7, 117-126 https://doi.org/10.1080/21641846.2019.1632614

Kent-Braun JA (1999): Central and peripheral contributions to muscle fatigue in humans during sustained maximal effort. Eur. J. Appl. Physiol. 80, 57-63 https://doi.org/10.1007/s004210050558
Kim W, Chang Y, Kim J, Seo J, Ryu K, Lee E, Janelle CM (2014): An fMRI study of differences in brain activity among elite, expert, and novice archers at the moment of optimal aiming. Cogn. Behav. Neurol. 27, 173-182 https://doi.org/10.1097/WNN.0000000000000042

Kujach S, Byun K, Hyodo K, Suwabe K, Fukuie T, Laskowski R, Dan I, Soya H (2018): A transferable high-intensity intermittent exercise improves executive performance in association with dorsolateral prefrontal activation in young adults. Neuroimage 169, $117-125$ https://doi.org/10.1016/j.neuroimage.2017.12.003

Meier J, Topka MS, Hänggi J (2016): Differences in cortical representation and structural connectivity of hands and feet between professional handball players and ballet dancers. Neural Plast. 2016 https://doi.org/10.1155/2016/6817397

Menant JC, Maidan I, Alcock L, Al-Yahya E, Cerasa A, Clark DJ, Mirelman A (2020): A consensus guide to using functional near-infrared spectroscopy in posture and gait research. Gait Posture 82, 254-265 https://doi.org/10.1016/j.gaitpost.2020.09.012

Milanovic Z, Sporis G, Weston M (2015): Effectiveness of highintensity interval training (HIT) and continuous endurance training for $\mathrm{VO} 2 \mathrm{max}$ improvements: A systematic review and meta-analysis of controlled trials. Sports Med. 45, 1469-1481 https://doi.org/10.1007/s40279-015-0365-0

Monroe DC, Gist NH, Freese EC, O`Connor PJ, McCully KK, Dishman RK (2016): Effects of sprint interval cycling on fatigue, energy, and cerebral oxygenation. Med. Sci. Sports. Exerc. 48, 615-624 https://doi.org/10.1249/MSS.0000000000000809

Naves JPA, Viana RB, Rebelo ACS, De Lira CAB, Pimentel GD, Lobo PCB, de Oliveira JC, Ramirez-Campillo R, Gentil P (2018): Effects of high-intensity interval training vs sprint interval training on anthropometric measures and cardiorespiratory fitness in healthy young women. Front. Physiol. 9, 1738 https://doi.org/10.3389/fphys.2018.01738

Paruk T, Rauch L, Jankiewicz M, Van Breda K, Stein D, King M (2020): Structural brain differences between ultra-endurance athletes and sedentary persons. Sports Med. Health Sci. 2, 89-94 https://doi.org/10.1016/j.smhs.2020.05.004

Pfurtscheller G, Bauernfeind G, Wriessnegger SC, Neuper C (2010): Focal frontal (de) oxyhemoglobin responses during simple arithmetic. Int. J. Psychophysiol. 76, 186-192 https://doi.org/10.1016/j.ijpsycho.2010.03.013

Pinti P, Scholkmann F, Hamilton A, Burgess P, Tachtsidis I (2018): Current status and issues regarding pre-processing of fNIRS neuroimaging data: An investigation of diverse signal filtering methods within a General Linear Model framework. Front. Hum. Neurosci. 12, 505 https://doi.org/10.3389/fnhum.2018.00505

Rypma B, Berger JS, D'esposito M (2002): The influence of workingmemory demand and subject performance on prefrontal cortical activity. J. Cogn. Neurosci. 14, 721-731 https://doi.org/10.1162/08989290260138627

Seidel O, Carius D, Roediger J, Rumpf S, Ragert P (2019): Changes in neurovascular coupling during cycling exercise measured 
by multi-distance fNIRS: a comparison between endurance athletes and physically active controls. Exp. Brain Res. 237, 2957-2972

https://doi.org/10.1007/s00221-019-05646-4

Seidel O, Ragert P (2020): Neurodiagnostics in sports: investigating the athlete's brain to augment performance and sport-specific skills. Front. Hum. Neurosci. 14, 133 https://doi.org/10.3389/fnhum.2020.00133

Škof B, Strojnik V (2006): Neuromuscular fatigue and recovery dynamics following prolonged continuous run at anaerobic threshold. Br. J. Sports Med. 40, 219-222 https://doi.org/10.1136/bjsm.2005.020966

Valenzuela PL, Maffiuletti NA, Joyner MJ, Lucia A, Lepers R (2019): Lifelong endurance exercise as a countermeasure against agerelated $\mathrm{V}^{\cdot} \mathrm{O} 2 \mathrm{max}$ decline: physiological overview and insights from masters athletes. Sports Med. 50, 703-716 https://doi.org/10.1007/s40279-019-01252-0

Vollaard NB, Metcalfe RS (2017): Research into the health benefits of sprint interval training should focus on protocols with fewer and shorter sprints. Sports Med. 47, 2443-2451 https://doi.org/10.1007/s40279-017-0727-x

von Lühmann A, Ortega-Martinez A, Boas DA, Yücel MA (2020): Using the general linear model to improve performance in fNIRS single trial analysis and classification: a perspective. Front. Hum. Neurosci. 14, 30 https://doi.org/10.3389/fnhum.2020.00030

Yücel MA, Lühmann AV, Scholkmann F, Gervain J, Dan I, Ayaz H, Wolf M (2021): Best practices for fNIRS publications. Neurophotonics 8, 012101

https://doi.org/10.1117/1.NPh.8.1.019802

Weinstein Y, Bediz C, Dotan R, Falk B (1998): Reliability of peaklactate, heart rate, and plasma volume following the Wingate test. Med. Sci. Sports Exerc. 30, 1456-1460 https://doi.org/10.1249/00005768-199809000-00017

Weir J, Beck T, Cramer J, Housh T (2006): Is fatigue all in your head? A critical review of the central governor model. Br. J. Sports Med. 40, 573-586 https://doi.org/10.1136/bjsm.2005.023028

Wiewelhove T, Raeder C, Meyer T, Kellmann M, Pfeiffer M, Ferrauti A (2015): Markers for routine assessment of fatigue and recovery in male and female team sport athletes during highintensity interval training. PloS One 10, e0139801 https://doi.org/10.1371/journal.pone.0139801

Wu J, Liu T, Huang J, Han W (2017): The effects of exercise fatigue on prefrontal and motor activations as measured by nearinfrared spectroscopy. Neuroreport 28, 1134-1138 https://doi.org/10.1097/WNR.0000000000000882

Received: April 16, 2021

Final version accepted: July 27, 2021 